Original Research Paper

\title{
Aetiology of Spontaneous Intracerebral Haemorrhage in Young Adults Admitted at a Tertiary Care Hospital in Dhaka
}

\author{
${ }^{1}$ Mohammad Enayet Hussain, ${ }^{2}$ Quazi Deen Mohammad, ${ }^{3}$ Monsur Habib, \\ ${ }^{4}$ Md. Azharul Hoque, ${ }^{5}$ Md. Badrul Alam and ${ }^{6}$ Md. Abdullah Yusuf \\ ${ }^{I}$ Department of Neurology, National Institute of Neurosciences and Hospital, Dhaka, Bangladesh \\ 2,4,5 Department of Neurology, National Institute of Neurosciences, Dhaka, Bangladesh \\ ${ }^{3}$ Department of Neurology, Dhaka Medical College, Dhaka, Bangladesh \\ ${ }^{6}$ Department of Microbiology, National Institute of Neurosciences, Dhaka, Bangladesh
}

Article history

Received: 17-07-2015

Revised: $20-08-2015$

Accepted: 11-09-2015

Corresponding Author: Mohammad Enayet Hussain Department of Neurology,

National Institute of

Neurosciences and Hospital,

Dhaka, Bangladesh

Email: enayetdmc@yahoo.com

\begin{abstract}
Spontaneous Intracerebral Haemorrhage (SICH) in young adult occurs due to multiple risk factors. The purpose of the present study was to determine the aetiological factors of spontaneous intracerebral haemorrhage in the young adult patients. This case control study was carried out in the Department of Neurology, Department of Neurosurgery and Department of Internal Medicine at Dhaka Medical College Hospital, Dhaka from September 2010 to August 2011 for a period of one (01) year. Patients aged 15 to 45 years who presented with spontaneous intracerebral haemorrhage were taken as case (Group A) and those more than 45 years of age with spontaneous intracerebral haemorrhage were taken as control (Group B). All the aetiological factors were recorded in a predesigned data sheet. A total of 430 patients with clinical feature of stroke were admitted through emergency and outpatient department during the study period, of which 152(35.34\%) patients had spontaneous intracerebral haemorrhage. Based on the inclusion and exclusion criteria 35 patients were recruited from 15-45 years age range as case and 25 patients were recruited as control. The risk factors of $\mathrm{SICH}$ in younger patients were arteriovenous malformation (45.7\%), toxaemia of pregnancy $(31.4 \%)$, hypertension $(17.1 \%)$, cavernous angioma $(2.9 \%)$, bleeding disorder $(2.9 \%)$ and coagulation disorder $(2.9 \%)$. In patients $>45$ years most of the ICH was due to HTN (52\%) followed by arteriovenous malformation $(20 \%)$. In conclusion arteriovenous malformation is the most common aetiological factors of young stroke patients followed by hypertension and toxaemia of pregnancy.
\end{abstract}

Keywords: Intracerebral Haemorrhage, Young Patients, Aetiology

\section{Introduction}

Spontaneous Intracerebral Hemorrhages (SICH) account for approximately $10 \%$ of all stroke cases (Mohr et al., 1978). The incidence of ICH in people aged $<35$ years has been estimated to be $0.3 / 100000$ (Wolf et al., 1992; Drury et al., 1984). The frequency of ICH among a series of stroke in young adults varies from 0.7 to $40 \%$ (Nencini et al., 1988; Bevan et al., 1990a). The etiologic spectrum of ICH in young people is also different than that in the older individuals and includes vascular malformations (Toffol et al., 1987a), hypertension (Fuh et al., 1994) and drug use (Caplan et al., 1982).
The 30-day case mortality of SICH is 40 to $50 \%$ (Broderick, 1994). Many of the patients who survive become disabled for the rest of their life. Whenever it occurs in younger persons, they may have a longer period of time to live with their disability and this increases the risk of other medical complications among these patients. The loss of productivity of a young person makes him a burden not only for his family but also for the country. Therefore, prevention remains the most important means of reducing its morbidity and mortality. Effective prevention requires understanding the aetiological factors of SICH and the population subgroups at greatest risk. While a specific 
definition of "young stroke" is lacking, the vast majority of authors consider "young stroke" pertaining to individuals less than 45 years of age. Different studies have included different age range ( 0 to 49 years) for study of intracerebral hemorrhage, but in most of the studies age group 15 to 45 years has been considered as the standard age group for the study of stroke. The aim of this study was to find out the underlying etiological factors in young patients (15-45 years) with spontaneous (i.e., non-traumatic) intracerebral hemorrhage.

\section{Materials and Methods}

This case control study was carried out in the Department of Neurology, Department of Neurosurgery and Department of Internal Medicine at Dhaka Medical College Hospital, Dhaka from September 2010 to August 2011 for a period of one (01) year. Patients with the age of 15 to 45 years with the clinical features of spontaneous intracerebral hemorrhage confirmed by CT-scan were taken as case (Group A) and more than 45 years of age with intracerebral haemorrhage confirmed by CT-scan were taken as control (Group B). Patients with traumatic intracerebral hemorrhage, subarachnoid hemorrhage and who were unwilling to take part were excluded from the study. Detail history was taken and thorough physical examination was performed in all patients by the investigator and findings were recorded. The information regarding age, sex, risk factors, clinical presentation were recorded on a data collection sheet. Then it was reviewed by a senior consultant neurologist before final diagnosis. Investigations like CBC, plasma glucose, BT, CT, Prothrombin time, fasting lipid profile, serum creatinine, urine $\mathrm{R} / \mathrm{E}$, serum electrolytes, ECG and chest X-ray were done. Among the special investigations MRA, CTA and DSA were done as appropriate. DSA was done only when neuroimaging like MRA or CTA failed to identify a suspected vascular malformation. The aetiology of $\mathrm{ICH}$ was defined in accordance with the criteria described by (Ruiz-Sandoval et al., 1999). Statistical analysis was performed by using SPSS version 12.0 (USA). Appropriate statistical methods were applied for data analysis and comparison. The significance of data was done with $95 \%$ confidence interval taking $\mathrm{p}$ value $<0.05$ as significant.

\section{Results}

Among 430 consecutive stroke patients admitted in the Neurology, Neurosurgery and Medicine department of Dhaka Medical College Hospital through emergency and outpatient department 152(35.3\%) patients had intracerebral hemorrhage. Among these 152 patients, 50(32.9\%) patients were in the 15-45 years of age group of which 2 patients died before the investigation for the aetiological search was completed, 9 patients were unwilling to participate in this study and 4 patients were unable to undergo the thorough investigations required for etiological search. Therefore the rest 35 patients were finally recruited in this study as case (group A). Again $102(67.1 \%)$ out of $152 \mathrm{ICH}$ patients were more than 45 years of which 21 patients died of various complications before etiological search was completed, 31 patients refused to undergo expensive investigations for etiological search and the rest 25 patients were unwilling to give the consent to participate in this study. Therefore the rest 25 patients were recruited as control (group B) for this study (Fig. 1).

The mean age of patients in group-I was $30.9 \pm 10.5$ years with range from 16 to 45 years and in group-II $59.6 \pm 9.2$ years with range 46 to 84 years. Maximum number of patients in group-I were found in the age group 16-25 years (15) and in group-II 56-65 years (11) (Table 1).

The most common causes of ICH in group I were rupture of an AVM in $16(45.7 \%)$ patients, HTN in 6 (17.1\%), toxaemia of pregnancy in $3(31.4 \%)$ out of 11 female patients. Cavernous angioma 1(2.9\%), bleeding disorder $1(2.9 \%)$ and coagulation disorder $1(2.9 \%)$ were among other causes of ICH. In group II the most common causes of $\mathrm{ICH}$ were HTN in $13(52 \%)$ patients whereas rupture of AVM was the cause of ICH in $5(20 \%)$ patients. In both groups cause remains unidentified in 7 patients (Group-I $20 \%$, group II 28\%) (Table 2).

Table 1. Age and Sex distribution of the study patients $(n=60)$

\begin{tabular}{llll}
\hline Variables & Group A & Group B & $P$ Value \\
\hline Age (mean \pm SD) & $30.9 \pm 10.5$ & $59.6 \pm 9.2$ & \\
Sex & & & \\
Male & $24(68.6 \%)$ & $16(64.0 \%)$ & 0.711 \\
Female & $11(31.4 \%)$ & $9(36.0 \%)$ & \\
\hline
\end{tabular}

$*$ male: female ratio $=2: 1$

Table 2. Aetiological factors of ICH among the study population

\begin{tabular}{llll}
\hline Aetiology & $\begin{array}{l}\text { Group A } \\
(\mathrm{n}=35)\end{array}$ & $\begin{array}{l}\text { Group B } \\
(\mathrm{n}=25)\end{array}$ & $P$ Value \\
\hline AVM & $16(45.7 \%)$ & $5(20.0 \%)$ & 0.039 \\
HTN & $6(17.1 \%)$ & $13(52.0 \%)$ & 0.004 \\
Cavernous angioma & $1(2.9 \%)$ & $0(0.0 \%)$ & 0.583 \\
Toxemia of pregnancy & $3(31.4 \%)$ & $0(0.0 \%)$ & 0.144 \\
Bleeding disorder & $1(2.9 \%)$ & $0(0.0 \%)$ & 0.583 \\
Coagulation disorder & $1(2.9 \%)$ & $0(0.0 \%)$ & 0.583 \\
Undetermined & $7(20.0 \%)$ & $7(28.0 \%)$ & 0.650 \\
\hline
\end{tabular}




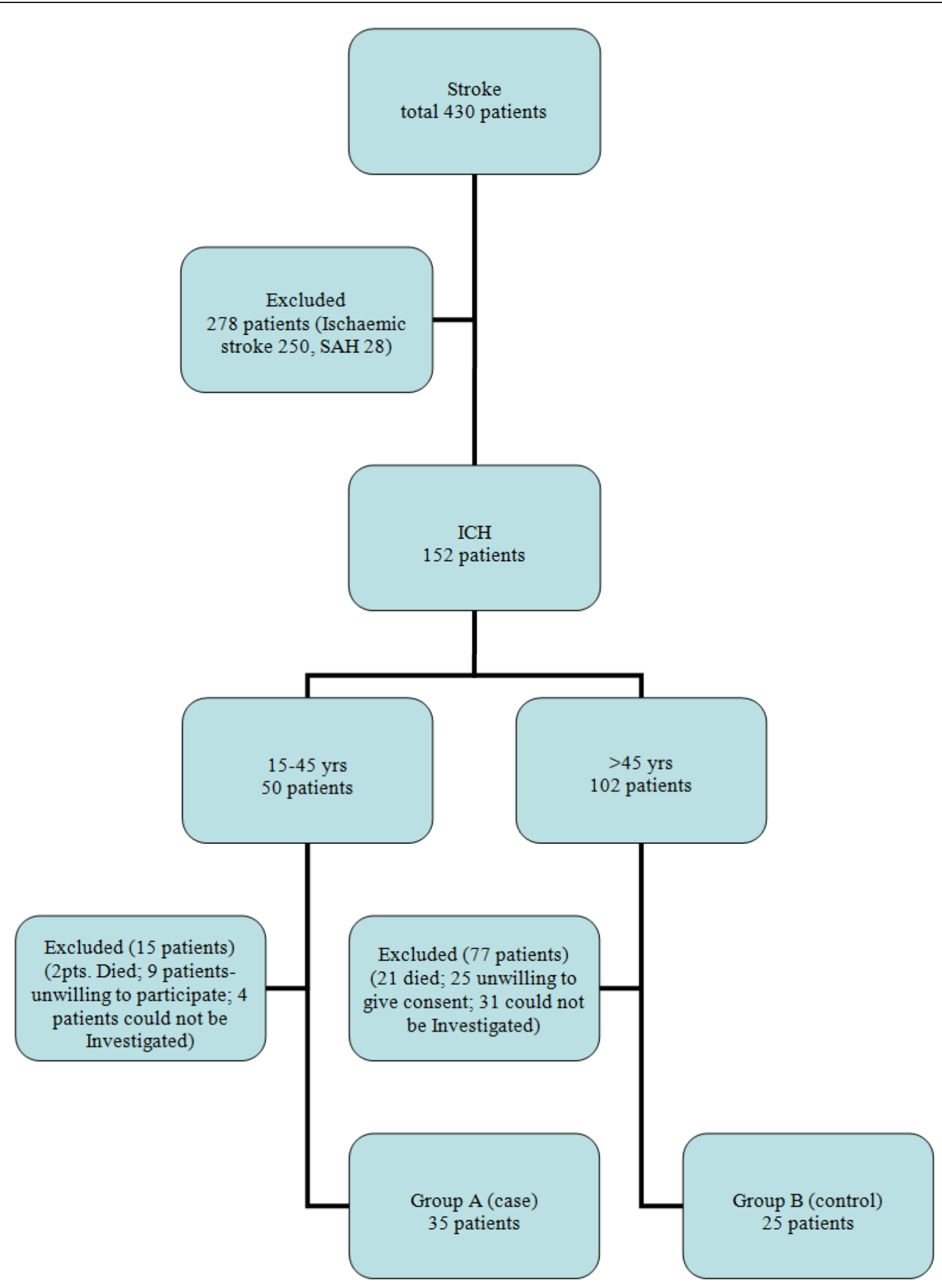

Fig. 1. Flow chart of the patients recruitment 


\section{Discussion}

In the current study the mean age of patients in group-I was $30.9 \pm 10.5$ and most (15) of the patients were in the age group 16-25. On the other hand the mean age of patients in group-II was $56.65 \pm 9.2$ and most patients (11) in this group were between 56-65 years. The male and female ratio in this study is almost $2: 1$ which is slightly higher than those reported from elsewhere (Bevan et al., 1990b). May be this is a reflection of low tendency of female patients for seeking medical advice in tertiary hospital rather relying on treatment of rural doctors as in other parts of the developing world.

In this study 6 different types of causes were found in the younger age group. Ruptured Arteriovenous Malformation (AVM) was found to be the most common (45.71\%) cause of spontaneous intracerebral hemorrhages. The other causes found in this group are hypertension (17.1\%), toxemia of pregnancy $3 / 11$ (31.4\%), cavernous angioma $(2.85 \%)$, bleeding disorder ( $2.85 \%)$, coagulation disorder $(2.85 \%)$. In $20 \%$ no cause could be determined. On the other hand in the older age group the most common cause was hypertension (52\%). Ruptured AVM was the cause of hemorrhage in $20 \%$. No cause could be determined in $28 \%$. So it is clear from the study that the most common cause of ICH in younger age group is vascular malformation particularly AVM ( $p=$ 0.039 ) whereas in the older age group it is hypertension ( $p$ $=0.004$ ). In this study no patient was found to have ICH due to drug abuse, vasculitis, Moyamoya disease because of the rarity of the diseases and probably also due to inclusion of a small number of patients.

Among 16 patients with ICH due to AVM in younger age group, $13(81.25 \%)$ were lobar in location. Rests 3 were located in the basal ganglia, brainstem and cerebellum. In the older age group out of $5,3(60 \%)$ were located in the lobar region and $2(40 \%)$ in the basal ganglia. Hayward (1976) found in his study that $57 \%$ of ICH secondary to a ruptured AVM was lobar. Ruiz-Sandoval et al. (1999) found among 67 patients with ICH and AVM, $45(67 \%)$ were lobar in location.

Hypertension as a cause of ICH was most common in individuals aged $>31$ years. In this study 5 of the 6 patients of ICH due to hypertension in group-I were of age $>40$ years, only one being the age of 32 years. Interestingly $2(33.3 \%)$ of the 6 patients were unaware of the presence of hypertension and in those in whom hypertension had been previously diagnosed (4), three were on irregular antihypertensive drug $(50 \%)$. In this group no secondary cause was found for hypertension. Ruiz-Sandoval et al. (1999) in their study of intracerebral hemorrhage in young people (15-40 years) found rupture of an AVM as the most common cause $(33 \%)$, followed by cavernous angioma (16\%), hypertension (11\%) which matches this study to some extent. Toffol et al. (1987b) in their study of nontraumatic intracerebral hemorrhage in 72 young adults (15-45 years) found ruptured AVM as the most common cause $(29.1 \%)$ of intracerebral hemorrhage, second being hypertension (15.3\%). In $23.6 \%$ the cause remained undetermined. Lin and Howng (1997) in their study of nontraumatic intracerebral hemorrhage in 91 young adult (15-40 years) found ruptured AVM and hypertension as the leading causes. They could not identify the underlying cause of hemorrhage in $25.3 \%$ patients. Lacy et al. (1984) reported in a study of stroke patients (17-44 years) where the identified causes were ruptured AVM, hypertension, sympathomimetic drug abuse and bacterial endocarditis. Hilton-Jones and Warlow (1982) reviewed their experience with 15 patients younger than 45 years who had nontraumatic ICH seen on CT. 5 probable causes like vascular malformation, hypertension, polyarteritisnodosa, bacterial endocarditis and hemophilia were identified. Hayward (1976) found that a non-traumatic ICH, seen on CT but not usually associated with aneurysmal rupture or primary hypertensive hemorrhage, is highly suggestive of an underlying AVM, particularly in a young patient. In his study, $57 \%$ cases of $\mathrm{ICH}$ secondary to a ruptured AVM were lobar. On the other hand hypertensive SICH occurred mostly in the gangliothalamic region which matches other study (Zafar and Khan, 2008).

In all the relevant studies on the etiology of spontaneous hemorrhage in young adults including the present study failed to determine the etiology in reasonably good number of patients. Several possible explanations can be found. Intracerebral hemorrhage could easily result from a ruptured "cryptic" AVM not detected by arteriography (Golden and Kramer, 1978; Terao et al., 1979). The true incidence of cryptic angiomas remains unknown. Biopsy of the hematoma and its wall appears to be the only way of obtaining valid diagnosis (Bitoh et al., 1982). Obliteration of a ruptured AVM or a saccular aneurysm may result from the primary event or hematoma. That is why some investigators recommend delaying angiography for several weeks for certain patients with otherwise unexplained ICH (Ropper and Davis, 1980). This study was based on data collected from Neurology, Neurosurgery and Medicine ward of a tertiary level hospital. As the sample size was small, the findings may not represent the picture in the population. Majority of the stroke patients are not referred to such a tertiary care hospital and only the more severe cases are admitted.

\section{Conclusion}

Although this is a small, hospital-based study, it reveals that young patients with spontaneous ICH represent a heterogenous group. The most common 
cause for spontaneous ICH in a young adult is a vascular malformation like ruptured AVM. The aetiology as well as the site of bleeding differs from those in the older group. Non-hypertensive causes are more common in younger age group unlike in the older age group where hypertension is the most common cause of ICH.

\section{Acknowledgement}

I am grateful to Dr Sharif Uddin Khan and Dr Kazi Mohibur Rahman who did the angiogram (DSA) in appropriate cases and to Dr Rajib Nayan Chowdhury for his valuable suggestions in preparing the manuscript.

\section{Funding Information}

The study was not funded by any authority.

\section{Author's Contributions}

Mohammad Enayet Hussain: Was involved in protocol preparation, data collection, literature search up to manuscript writing.

Quazi Deen Mohammad, Monsur Habib, Md. Azharul Hoque, Md. Badrul Alam: Were involved in manuscript preparation.

Md. Abdullah Yusuf: Was involved in statistical analysis.

\section{Ethics}

Informed written consent was taken from all patients. Ehical approval for the study was obtained from the Ethical review committee of Dhaka Medical College.

\section{References}

Bevan, H., K. Sharma and W. Bradley, 1990a. Stroke in young adults. Stroke, 21: 382-386. DOI: 10.1161/01.STR.21.3.382

Bevan, H., K. Sharma and W. Bradley, 1990b. Stroke in young adults. Stroke, 21: 382-386. DOI: 10.1161/01.STR.21.3.382

Bitoh, S., H. Hasegawa, M. Fujiwara and M. Sakurai, 1982. Angiographically occult vascular malformations causing intracranial hemorrhage. Surg. Neurol., 17: 35-42. DOI: 10.1016/0090-3019(82)90039-8

Broderick, J., 1994. Intracerebral Hemorrhage. In: Handbook of Neuroepidemiology, Gorelick, P. and M. Alter (Eds.), NY: Marcel Dekker Inc, New York, ISBN-10: 0824792424, pp: 141-167.

Caplan, L., D.B. Hier and G. Banks, 1982. Current concept of cerebrovascular disease-stroke: Stroke and drug abuse stroke and drug abuse. Stroke, 13: 869-872. DOI: 10.1161/01.STR.13.6.869
Drury, I., J.P. Whisnant and W.M. Garraway, 1984. Primary intracerebral hemorrhage: Impact of $\mathrm{CT}$ on incidence. Neurology, 34: 653-657.

DOI: 10.1212/WNL.34.5.653

Fuh, J.L., H.C. Liu, S.J. Wang, Y.K. Lo and L.S. Lee, 1994. Nontraumatic hemorrhagic stroke in young adults in Taiwan. J. Stroke, Cerebrovasc. Dis., 4: 101-105. DOI: 10.1016/S1052-3057(10)80117-X

Golden, J.B. and R.A. Kramer, 1978. The angiographically occult cerebrovascular malformation report of three cases. J. Neurosurg, 48: 292-296. DOI: 10.3171/jns.1978.48.2.0292

Hayward, R.D., 1976. Intracranial arteriovenous malformations. Observations after experience with computerised tomography. J. Neurol. Neurosur. Psychiatry, 39: 1027-1033. DOI: 10.1136/jnnp.39.10.1027

Hilton-Jones, D. and C.P. Warlow, 1982. The causes of stroke in the young. J. Neurol., 232: 137-143. DOI: $10.1007 /$ BF00313888

Lacy, J.R., C.M. Filley, M.P. Earnest and N.R. GraffRadford, 1984. Brain infarction and hemorrhage in young and middle-aged adults. West J. Med., 141: 329-334.

Lin, C.L. and S.L. Howng, 1997. Nontraumatic intracerebral hemorrhage in young adult. Kaohsiung J. Med. Sci., 13: 237-242. PMID: 9177085

Mohr, J.P., L.R. Caplan, J.W. Melski, R.J. Goldstein and G.W. Duncan et al. 1978. The harvard cooperative stroke registry: A prospective registry. Neurology, 28: 754-762. DOI: 10.1212/WNL.28.8.754

Nencini, P., D. Inzitari, M.C. Baruffi, L. Fratiglioni and R. Gagliardi et al., 1988. Incidence of stroke in young adults in Florence, Italy. Stroke, 19: 977-981. DOI: $10.1161 / 01 . S T R .19 .8 .977$

Ropper, A.H. and K.R. Davis, 1980. Lobar cerebral hemorrhages: Acute clinical syndromes in 26 cases. Ann. Neurol., 8: 141-147. DOI: $10.1002 /$ ana.410080203

Ruiz-Sandoval, J.L., C. Cantu and F. Barinagarrementeria, $1999 . \quad$ Intracerebral hemorrhage in young people: Analysis of Risk Factors, Location, Causes and Prognosis. Stroke, 30: 537-541. DOI: 10.1161/01.STR.30.3.537

Terao, H., T. Hori, M. Matsutani and R. Okeda, 1979. Detection of cryptic vascular malformations by computerized tomography report of two cases. J. Neurosurg, 51: 546-551. DOI: $10.3171 /$ jns. 1979.51 .4 .0546

Toffol, G.J., J. Biller and H.P. Adams, 1987a. Nontraumatic intracerebral hemorrhage in young adults. Arch. Neurol., 44: 483-485. DOI: 10.1001/archneur.1987.00520170013014

Toffol, J.C., J. Biller and H.P. Adams, 1987b. Nontraumatic intracerebral hemorrhage in young adults. Arch. Neurol., 44: 483-485. DOI: 10.1001/archneur.1987.00520170013014 
Wolf, P.A., J.L. Cobb and R.B. Agostino, 1992. Epidemiology of Stroke. In: Stroke: Pathophysiology, Diagnosis and Management, H.J.M. Barnett, J.P. Mohr, B. Stein, F. Yatsu (Eds.), NY: Churchill Livingstone, New York, pp: 6-6.
Zafar, A. and F.S. Khan, 2008. Clinical and radiological features of intracerebral haemorrhage in hypertensive patients. J. Pak. Med. Assoc., 58: 356-358. PMID: 18988404 